\title{
Childhood Autism Spectrum Disorder and Epilepsy Co-occurrence: Sub- population Prevalence Variances and Risk Modeling
}

\section{Larry Holmes Jr. ${ }^{1,2,3^{*}}$, Leslie Stalnaker ${ }^{1,4}$, Joseph Casini ${ }^{1,5}$, Isabel Morgan ${ }^{1,6}$, Valescia John ${ }^{1}$ and Kirk Dabney ${ }^{1}$}

${ }^{1}$ Nemours/Alfred I. DuPont Hospital for Children, Office of Health Equity \& Inclusion, Health Disparities Science Research, Wilmington, DE, USA

${ }^{2}$ Biological Sciences Department, University of Delaware, Newark, DE, USA

${ }^{3}$ Medical College of Wisconsin, Milwaukee, WI, USA

${ }^{4}$ Department of Public \& Community Health, Liberty University, Lynchburg, VA, USA

${ }^{5}$ Villanova University, Villanova, PA, USA

6Johns Hopkins Bloomberg School of Public Health, Baltimore, MD, USA

"Corresponding author: Holmes LJ, Office of Health Equity \& Inclusion, Nemours/Alfred I. DuPont Hospital for Children, P.O. Box 269, Wilmington, DE, USA, 19899; Email: Imholmesjr@gmail.com

Receiving date: October 27, 2017, Acceptance date: November 15, 2017, Published date: November 29, 2017

Copyright: (c) 2017 Holmes LJ, et al. This is an open-access article distributed under the terms of the Creative Commons Attribution License, which permits unrestricted use, distribution, and reproduction in any medium, provided the original author and source are credited.

\begin{abstract}
Autism spectrum disorder (ASD) tends to co-occur with epilepsy, but it remains unclear if ASD predisposes to epilepsy or vice versa. Using the National Survey of Children's Health (2012), we assessed the relationship between ASD and epilepsy as disease co-occurrence, examined racial/ethnic variability therein and the risk markers. Compared to whites, blacks/African Americans were $54 \%$ less likely to be diagnosed with ASD, prevalence odds ratio $(P O R)=0.46,95 \% \mathrm{Cl}, 0.34-0.63$. In contrast, compared to whites, blacks/African Americans were $56 \%$ more likely to be diagnosed with epilepsy, POR=1.56, 95\% Cl, 1.24-1.96. Similarly, racial differences were observed in cooccurrence, with significant $20 \%$ higher risk among Blacks relative to Whites, $P O R=1.20, p<0.0001$. Public insurance, poverty, maternal education, and intellectual disabilities were the two most potent predictors of ASDepilepsy co-occurrence. After controlling for age, sex, parental education, insurance coverage, federal poverty level, and intellectual disability, significant racial differences did not persist but Blacks were $74 \%$ less likely to report ASD \& epilepsy co-occurrence relative to Whites, $\mathrm{aPOR}=0.26, p=0.23$. In summary, racial disparities exist in ASD-epilepsy co-occurrence prevalence, which did not persist after controlling for potential confounders; while public insurance, maternal education, poverty and intellectual disabilities remain potent predictors of the co-occurrence. These findings suggestive of cautious optimism in assessing the causal direction in the co-occurrence of ASD and epilepsy among children.
\end{abstract}

Keywords: Autism; Autism Spectrum Disorder; Epilepsy; AutismEpilepsy Co-occurrence; Race; Pediatrics; Childhood; Risk modeling

\section{Introduction}

Autism spectrum disorder (ASD) is a range of neurodevelopmental disorders characterized by the broad autism phenotype (BAP) which demonstrates impairments in communication, difficulty in social interactions, and stereotyped patterns of behaviors, interests and activities [1,2]. Although, this condition is largely misunderstood, recent epidemiological evidence suggests that by age 8,1 out of 88 children will be diagnosed with ASD, with more diagnoses being reported each year $[3,4]$. The pathophysiology of ASD has been difficult to identify due to the uncertainty surrounding its origins. The early overgrowth of higher cognitive brain regions in development is believed to contribute to an imbalance between excitatory-inhibitory networks, which is reflected by ASD phenotypes such as child agitation and stereotyped behaviors [5]. These phenotypes underscore the frequent comorbidities of ASD with Fragile X syndrome, tuberous sclerosis, Tourette syndrome, attention deficit disorder and epileptic seizure [6].

Epilepsy is a chronic disease identified as a manifestation of abnormal, excessive hypersynchronous firing of cortical neurons [6]. It has been reported to be one of the most prevalent neurological disorders, affecting approximately $0.6 \%$ of children ages seventeen or younger; while varying in degree of severity, epilepsy impacts over 50 million people globally $[7,8]$. Similarly to ASD, there has been ambiguity in identifying its origins and pathophysiology. Bromfield et al. propose that a combination of action potentials decreased inhibitory neurotransmission, and alterations of intra or extra-cellular ion concentrations result in membrane depolarization [6]. Such action potentials in association with overgrown axonal collaterals are capable of initiating self-reinforcing circuits that extend instances of excitation and ultimately present as epileptic seizure [6].

Increased attention to these chronic diseases, particularly ASD, has revealed higher prevalence than has been previously recognized. Methodological changes in clinical diagnoses have seen drastic rises in ASD incidence, namely from 1 in 150 diagnosed children in 2000 to 1 in 68 diagnosed children in 2010 [9]. Furthermore, several studies have suggested that $5 \%$ to $46 \%$ of individuals diagnosed with ASD have been observed to develop some degree of epilepsy, with the greatest risk of development appearing in adolescence [10-12]. Previous research studying ASD revealed that males were more likely to be diagnosed by a ratio of 4:1, a finding that has been comparable to other neurodevelopmental comorbidities such as dyslexia and attention deficit disorder $[1,13]$. 
To our knowledge, there have been very few studies assessing the co-occurrence of ASD and epilepsy, which makes it difficult to establish valid and reliable prevalence of this co-occurrence. With respect to symptoms and manifestations, there are similarities between the two conditions, yet no causal relationships between ASD and epilepsy have been established suggesting that ASD may be a contributing cause to epilepsy or vice versa. While previous data have explained the presence of co-occurring ASD and epilepsy, there remains a pressing need for more research [1,4,10-13]. Evaluation of studies on the prevalence of epilepsy and ASD illustrates the challenges in obtaining reliable and accurate prevalence in these conditions. Preliminary studies on disease origins, overlapping phenotypes, and frequency of comorbidity have provoked increased speculation towards an association between ASD and epilepsy [4,10-14]. Relative to the general population, epilepsy is more common in children with ASD (prototypical form of a group of pervasive developmental disorders that comprise autism, atypical autism, Asperger syndrome and 'other' pervasive development disorder) [4,10-13]. While an association between these two clinical conditions has been established, shared features including phenotypes remain to be fully understood as well as the causal relationship in terms of infantile seizure's etiologic role in ASD. Additionally, it is unknown if the prevalence of the comorbidity or relationship of these two conditions varies by race and ethnicity, and if so, which risk markers or factors explain such variance.

In this study, we aimed to examine the prevalence of ASD, epilepsy and co-occurrence, a broad term implying the presence of both clinical conditions (ASD and epilepsy). Additionally, we sought to identify the risk markers for dual diagnosis among the sample with either of the conditions relative to those with both conditions.

\section{Materials and Methods}

We conducted a cross-sectional study to examine the prevalence of ASD, epilepsy and the co-occurrence of these conditions and to examine potential variance or relationships by race, insurance coverage, and other risk markers. The secondary data were provided by the United States' National Survey of Children's Health (NSCH), 2012, which is a cross-sectional survey of non-institutionalized residents. An Internal Review Board (IRB) approval was not required for this study due to utilization of public access data (NSCH, 2011/12).

\section{Study population and sample}

The study population $(\mathrm{n}=95,775)$ consisted of non-institutionalized children aged 0-17 in the United States' National Survey of Children's Health, 2011/12. The racial distribution of children in the sample was white $\quad(n=68,738,73.8 \%)$ Black $(n=9,493,10.2 \%)$ and other $(\mathrm{n}=14,886,16.0 \%)$. All children meeting this eligibility criterion were included regardless of race, ethnicity, sex, or other stratifying characteristics. Parents or heads of household, including caregivers, were asked a series of questions regarding the child's health and healthcare history, family demographics, and living environment. For the purposes of this study, parents/caregivers were asked to report whether or not the child had ever been diagnosed with epilepsy or if the child had ever been diagnosed with autism, Asperger's disorder, or pervasive development disorder. Based on these responses, the sample size utilized was 2,072. This sample size is representative of the population of children in the United States and is considered reliable for interpretation and generalization.

\section{Study variables ascertainment}

Study variables consisted of self-reported conditions of ASD and epilepsy as well as other self-reported characteristics including race, age, sex, federal poverty level, health insurance coverage, and intellectual disability, maternal and paternal education. In order to facilitate data analysis, ASD and epilepsy variables were processed and recoded to create binary variables $(0=$ absence, $1=$ presence). The race variable in this study focused primarily on 'white' and 'black' participants as well as 'other', those who identified as another race category other than white or black. To better assess ASD, epilepsy, and co-occurrence by age, the continuous age variable was recoded, creating age categories of '2-5 years, ' $6-9$ years', ' $10-14$ years' and ' $15-17$ years. This categorical age distribution allowed us to narrow our findings in a way that accounts for degrees of maturity and developmental and behavioral differences in the pediatric population. Sex, a dichotomous variable, indicated children as male or female on a nominal scale. Poverty level was utilized to demonstrate relative socioeconomic status with $100 \%$ equaling the federal poverty level. This variable was recoded and categorized into ' $0-199 \%$ federal poverty level (FPL) and ' $200 \%$ or greater FPL' Additionally, we utilized a categorical variable for health insurance coverage, measured as 'Public/ Medicaid', 'Private', or 'Uninsured'. Intellectual disability was used to identify whether or not the children currently have or were ever diagnosed with intellectual disability or mental retardation. Intellectual disability was represented as a categorical variable characterized as 'No condition', 'Ever told, no current condition' and 'Current condition'. 'Maternal education' and 'paternal education' were included in the study to explore the impacts of parental education levels on ASD, epilepsy, and co-occurrence. Education variables were measured in three categories: 'Less than high school', 'High school graduate,' and 'More than high school'. Body mass index (BMI) was also utilized to examine relationships between ASD and epilepsy. BMI was measured as 'Less than $5 \%$,' ' $5 \%-<85 \%$ ', ' $85 \%-<95 \%$ 'and “ $95 \%$ or greater.' The rationale for including $\mathrm{BMI}$ as one of the measures of the cooccurrence results from the perpetual association observed between adiposity, total body fat, and morbidities as well as co-occurrence. Despite the fact that BMI does not accurately assess adipocytes and body fats that have been implicated in most pathology, it remains a proxy to adipocytes.

\section{Statistical analysis}

Data processing was performed to examine missing variables and outliers. The categorical and nominal variables were summarized using frequency and percentages. The probability or likelihood of having been told that a child has ASD, epilepsy or co-occurrence was examined using a positive response as a numerator divided by the population at risk as a denominator, implying a simple probability of an event occurring or not occurring as the prevalence of the condition. Additionally, the co-occurrence of ASD and epilepsy was assessed using prevalence odds ratio (POR) estimates, given race. In order to determine whether or not there was co-occurrence among children with ASD and children with epilepsy, we utilized Pearson Chi-square statistic as well as univariable and multivariable logistic regression models.

The multivariable logistic regression model was used to examine cooccurrence given race, sex and predictors, as well as potential confounding variables. Prior to building a multivariable model, we assessed variables for effect measure modification and confounding effects using Mantel-Haenszel stratification analysis. A multivariable 
logistic regression model was built to examine the simultaneous effect of race, sex, age, maternal and paternal education, poverty, insurance coverage, and intellectual disability on ASD and epilepsy cooccurrence. Forward loading and backward elimination were utilized to construct the model. The variables that illustrated at least $10 \%$ difference between the crude and adjusted estimate were included as confounding in the multivariable model. All tests were two-tailed in the hypothesis testing model with $\mathrm{p}$ value method. The significance level for the univariable model was 0.05 ( $95 \%$ confidence interval), while the multivariable model was 0.01 ( $99 \%$ confidence interval). The entire analyses were performed using Stata version 14 [15].

\section{Results}

The sample for the assessment of the prevalence of ASD, epilepsy and co-occurrence od ASD and epilepsy was 95, 677 and comprised white $(n=68,736)$, black $(n=9,493)$, and other $(n=14,886)$. Although not shown in the tables, the prevalence of epilepsy was $n=579(0.7 \%)$, ASD, $\mathrm{n}=1,624$ (1.9\%) and co-occurrence, $\mathrm{n}=101 \quad(0.12 \%)$. The prevalence of ASD among whites was $1.8 \%(1,232)$, blacks, $132(1.4 \%)$, and other, $233(1.6 \%)$. The seizure prevalence was $0.6 \%$ (428) among whites, $1.0 \%$ (92) among blacks and $0.6 \%$ (86) among other. The prevalence of ASD and epilepsy co-occurrence was $0.11 \%$ (76) among whites, $0.13 \%$ (12) among blacks and $0.9 \%$ (14). There were racial disparities in epilepsy, with prevalence higher among blacks (1.0\%) relative to whites $(0.7 \%) \times 2(2)=15.7, \mathrm{p}<0.001$. However, ASD was more prevalent among whites $(2.0 \%)$, lowest among blacks (1.6\%) and intermediate among other race (1.8\%), $\chi^{2}(2)=10.2, \mathrm{p}<0.01$.

\section{Study characteristics for ASD, epilepsy, and co-occurrence stratified by race}

Although not shown in the tables, variance was observed in the study characteristics of children with autistic spectrum disorder (ASD) stratified by race. There were racial variances in ASD by age. While ASD was comparable between races, the prevalence was highest in age group 10-14 years. Regarding health insurance, African Americans (33.6\%) were less likely to have private insurance compared to their white $(55.2 \%)$ counterparts, $\chi^{2}(4)=30.1, \mathrm{p}<0.001$. African Americans/ blacks $(30.5 \%)$ were more likely to present with intellectual disability compared to whites $(18.7 \%), \chi^{2}(4)=14.6, p=0.01$. There were racial differences in mother's education, with African American mothers (71\%) slightly less likely to have higher than high school diploma relative to whites $(76 \%), \chi^{2}(4)=12.44, p=0.01$. Similarly, African American fathers (62.9\%) were less likely compared to whites $(73.3 \%)$ to have more than high school diploma, $\chi^{2}(4)=9.8, p=0.04$. The body mass index (BMI) 95\% was more common among blacks $(37.7 \%)$ compared to whites $(21.5 \%), \chi^{2}(6)=13.1, \mathrm{p}=0.04$. There were no racial variances in the sex of children with ASD.

Similar to ASD, racial variance was demonstrated in the study characteristics of children with epilepsy. There were racial differences in regards to federal poverty level, with whites $(57.5 \%)$ more likely to be in the upper level of income ( $\geq 200 \mathrm{FPL}$ ) when compared to their black counterparts $(27.3 \%), \chi^{2}(2)=26.7, \mathrm{p}<0.001$. There was also a marginally significant racial difference in health insurance, with whites $(42.1 \%)$ more likely to have private coverage relative to black/African Americans (26.1\%), $\chi 2$ (4)=7.9, p=0.09. There were no significant racial differences in intellectual disability, mother's education and body mass index.
The study characteristics of children with both ASD and epilepsy, broadly termed co-occurrence in this study, implying the presence of two clinical conditions also demonstrated racial variance. Health insurance coverage and federal poverty level indicated significant racial differences. Among children with co-occurrence, there was racial variability in the distribution of public insurance comparing white $(60.5 \%)$ to black (91.7\%) children. Similarly, Blacks/African Americans (16.7\%) were less likely to be classified with $\geq 200 \%$ FPL in the poverty category when relative to whites $(60.5 \%)$.

\section{Crude association between ASD, epilepsy, co-occurrence and race}

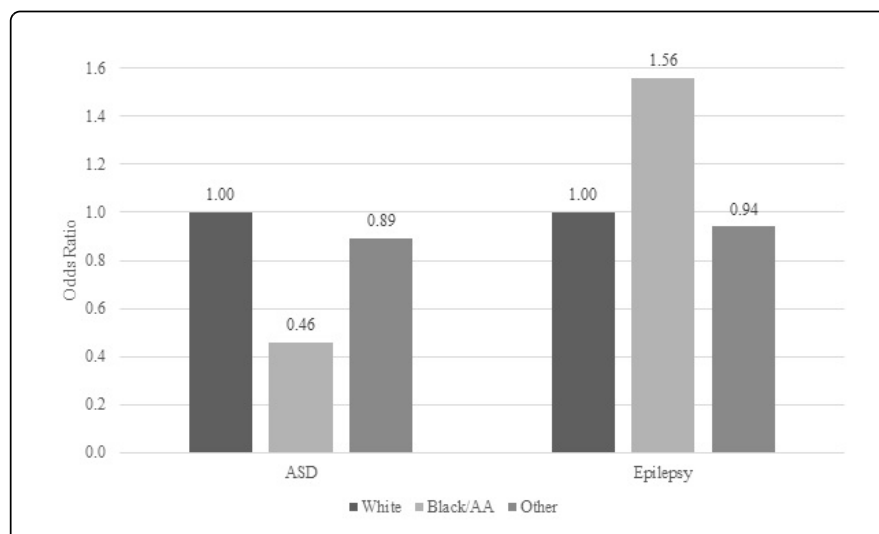

Figure 1: Unadjusted association between race and Autism Spectrum Disorder and Epilepsy, National survey of children's health, 2012.

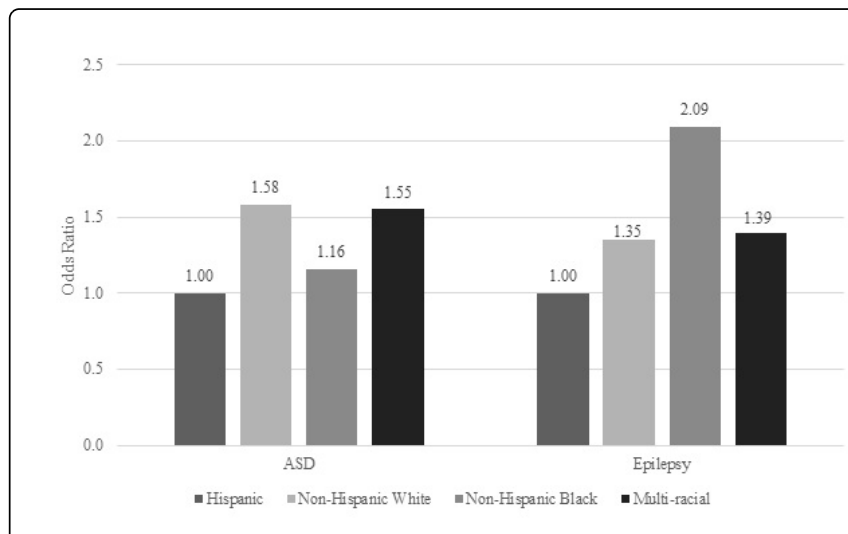

Figure 2: Unadjusted association between ethnicity and Autism Spectrum Disorder and Epilepsy, National survey of children's health, 2012.

Table 1 presents the crude association between race, and cooccurrence as well as ASD and epilepsy using univariable logistic regression. Whereas African Americans/blacks were less likely to be diagnosed with ASD, they were more likely to be diagnosed with epilepsy and co-occurrence. Compared to whites, blacks/African Americans were $54 \%$ less likely to be diagnosed with ASD, prevalence odds ratio $(\mathrm{POR})=0.46,95 \% \mathrm{CI}, 0.34-0.63$. In contrast, compared to whites, blacks/African Americans were 56\% more likely to be diagnosed with epilepsy, $\mathrm{POR}=1.56,95 \% \mathrm{CI}, 1.24-1.96$. Although 
Citation: Holmes LJ, Stalnaker L, Casini J, Morgan I, John V, et al. (2017) Childhood Autism Spectrum Disorder and Epilepsy Co-occurrence: Sub-population Prevalence Variances and Risk Modeling. Epilepsy J 3: 1000119. doi:10.4172/2472-0895.1000119

Page 4 of 8

imprecise, African Americans/blacks were $20 \%$ more likely to be diagnosed with both conditions relative to whites, $\mathrm{POR}=1.20$, 95\% CI, 0.64-2.25. The sample estimates (ORs) by race is illustrated in Figure 1, indicative of higher ASD among whites, while higher epilepsy was observed among blacks, Figure 2 presenting the unattested association by ethnicity.

\begin{tabular}{|c|c|c|c|c|c|c|c|c|c|}
\hline \multirow{2}{*}{ Variable } & \multicolumn{3}{|l|}{ ASD } & \multicolumn{3}{|c|}{ Epilepsy } & \multicolumn{3}{|c|}{ Co-occurrence } \\
\hline & OR & $95 \% \mathrm{Cl}$ & $\mathbf{P}$ & OR & $95 \% \mathrm{Cl}$ & $\mathbf{P}$ & OR & $95 \% \mathrm{Cl}$ & $\mathbf{P}$ \\
\hline \multicolumn{10}{|l|}{ Race } \\
\hline White & 1 & Referent & Referent & 1 & Referent & Referent & 1 & Referent & Referent \\
\hline Black & 0.46 & $0.34-0.63$ & $<0.001$ & 1.56 & $1.24-1.96$ & 0.002 & 1.2 & $0.64-2.25$ & 0.56 \\
\hline Other & 0.89 & $0.66-1.20$ & 0.89 & 0.94 & $0.74-1.19$ & 0.626 & 0.89 & $0.49-1.63$ & 0.71 \\
\hline
\end{tabular}

Table 1: Association between race and ASD, Epilepsy, and ASD/Epilepsy Co-occurrence, National survey of children's health, 2012.

Notes and abbreviations: This table represents the unadjusted association calculated through logistic regression. The type 1 error tolerance [significance level] was set at 5\% [0.05]. or Odds Ratio; \%, percentage; CI, Confidence Interval; P, p-value. The adjusted prevalence odds ratio for the group 'Uninsured' was omitted due to an insufficient sample.

\section{Crude association between co-occurrence and other study characteristics}

Table 2 exhibits the univariable and unadjusted regression model of risk and predisposing factors in ASD-Epilepsy co-occurrence. There were significant differences in health insurance and intellectual disability with respect to co-occurrence (ASD and epilepsy). Children who currently had intellectual disability were 4 times as likely to have been diagnosed with ASD and epilepsy (co-occurrence), $\mathrm{POR}=4.22$, 95\% CI, 2.81-6.34. Likewise, compared to children with public insurance, those with private insurance were $45 \%$ less likely to be diagnosed with co-occurrence, $\mathrm{POR}=0.55,95 \% \mathrm{CI}, 0.36-0.38$. The univariable association between the potential predictors of the cooccurrence is illustrated in Figure 3 as risk markers, namely intellectual disability, insurance and poverty.

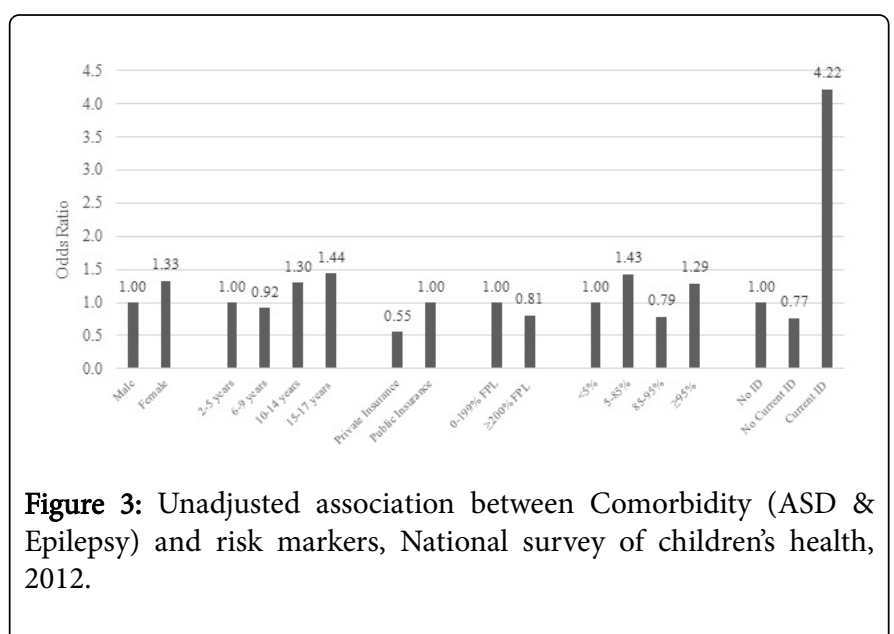

\begin{tabular}{|c|c|c|c|}
\hline Variable & POR & $95 \% \mathrm{Cl}$ & $\mathbf{P}$ \\
\hline \multicolumn{4}{|l|}{ Sex } \\
\hline Male & 1 & \multicolumn{2}{|l|}{ Reference } \\
\hline Female & 1.33 & $0.86-2.06$ & 0.193 \\
\hline \multicolumn{4}{|l|}{ Race } \\
\hline White & 1 & \multicolumn{2}{|l|}{ Reference } \\
\hline Black & 1.2 & $0.64-2.25$ & 0.564 \\
\hline Other & 0.89 & $0.39-1.63$ & 0.705 \\
\hline \multicolumn{4}{|l|}{ Ethnicity } \\
\hline Hispanic & 1 & \multicolumn{2}{|l|}{ Reference } \\
\hline Non-Hispanic White & 1.36 & $0.62-3.00$ & 0.445 \\
\hline Non-Hispanic Black & 1.76 & $0.68-4.57$ & 0.245 \\
\hline Multi-racial & 1.41 & $0.54-3.66$ & 0.479 \\
\hline
\end{tabular}


Citation: Holmes LJ, Stalnaker L, Casini J, Morgan I, John V, et al. (2017) Childhood Autism Spectrum Disorder and Epilepsy Co-occurrence: Sub-population Prevalence Variances and Risk Modeling. Epilepsy J 3: 1000119. doi:10.4172/2472-0895.1000119

Page 5 of 8

\begin{tabular}{|c|c|c|c|}
\hline Age & & & \\
\hline $2-5$ years & 1.00 & Reference & \\
\hline $6-9$ years & 0.92 & $0.45-1.86$ & 0.818 \\
\hline $10-14$ years & 1.3 & $0.68-2.45$ & 0.425 \\
\hline $15-17$ years & 1.44 & $0.73-2.85$ & 0.292 \\
\hline Federal Poverty Level & & & \\
\hline 0-199\% FPL & 1 & Reference & \\
\hline$\geq 200 \mathrm{FPL}$ & 0.81 & $0.54-1.21$ & 0.303 \\
\hline Health Insurance Coverage & & & \\
\hline Public/Medicaid & 1 & Reference & \\
\hline Private & 0.55 & $0.36-0.83$ & 0.004 \\
\hline Uninsured & Omitted & & \\
\hline Intellectual Disability & & & \\
\hline No condition & 1.00 & Reference & \\
\hline Ever told, no current condition & 0.77 & $0.10-5.75$ & 0.804 \\
\hline Current condition & 4.22 & $2.81-6.34$ & $<0.001$ \\
\hline Maternal Education & & & \\
\hline Less than high school & 1 & Reference & \\
\hline High school graduate & 0.82 & $0.33-2.02$ & 0.664 \\
\hline More than high school & 0.9 & $0.40-2.00$ & 0.799 \\
\hline Paternal Education & & & \\
\hline Less than high school & 1 & Reference & \\
\hline High school graduate & 2.59 & $0.59-11.40$ & 0.21 \\
\hline More than high school & 2.01 & $0.48-8.46$ & 0.34 \\
\hline Body Mass Index (BMI) & & & \\
\hline$<5 \%$ & 1 & Reference & \\
\hline $5 \%-<85 \%$ & 1.43 & $0.50-4.10$ & 0.51 \\
\hline $85 \%$ - <95\% & 0.79 & $0.23-2.79$ & 0.72 \\
\hline$\geq 95 \%$ & 1.29 & $0.42-3.96$ & 0.66 \\
\hline
\end{tabular}

Table 2: Univariable association between sex, race, and other risk markers and co-occurrence (ASD \& Epilepsy), National survey of children's health, 2012.

Notes and Abbreviations: The type 1 error tolerance [significance level] was set at 5\% [0.05]. POR, prevalence odds ratio; n, number; \%, percentage; FPL, federal poverty line; CI, Confidence Interval; P, p-value. The adjusted prevalence odds ratio for the group 'Uninsured' was omitted due to an insufficient sample.

\section{Adjusted association between co-occurrence and race}

Table 3 indicates the effect of race on co-occurrence after adjusting for various biologically and socially relevant variables as confounding. After controlling for age and sex, as biologically relevant variables in the disease process in Model I, the risk increased only slightly for both blacks and others. However, after adjusting for federal poverty level, maternal education, and paternal education in Model II, the racial differences was modified. Blacks/AA were approximately $72 \%$ less likely to have co-occurrence relative to whites, adjusted POR (aPOR $)=0.28,99 \% \mathrm{CI}, 0.02,3.85, \mathrm{p}=0.21$. After controlling for other confounding variables in Models III and IV, such as insurance coverage and intellectual disability, the association did not change significantly, 
Citation: Holmes LJ, Stalnaker L, Casini J, Morgan I, John V, et al. (2017) Childhood Autism Spectrum Disorder and Epilepsy Co-occurrence: Sub-population Prevalence Variances and Risk Modeling. Epilepsy J 3: 1000119. doi:10.4172/2472-0895.1000119

Page 6 of 8

and racial differences did not persisted, but Blacks/AA were $74 \%$ less likely to report of ASD \& epilepsy co-occurrence relative to Whites, $\mathrm{aPOR}=0.26, \mathrm{p}=0.23$. Figure 4 indicates the adjusted and unadjusted point estimates on the association between race and co-occurrence as comorbidities. The adjusted and unadjusted association is illustrated in Figure 5.

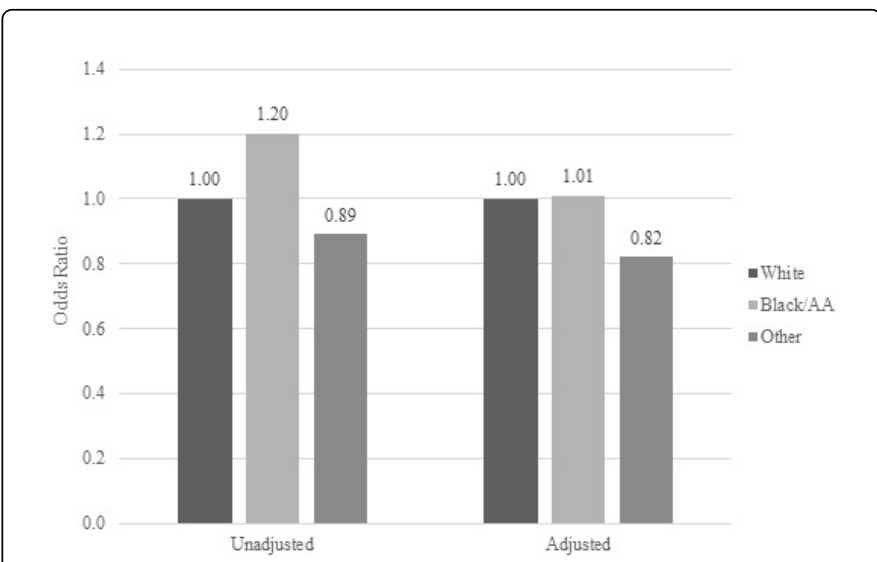

Figure 4: Unadjusted and adjusted associations between race and comorbidity (ASD and Epilepsy), National survey of children's health, 2012.

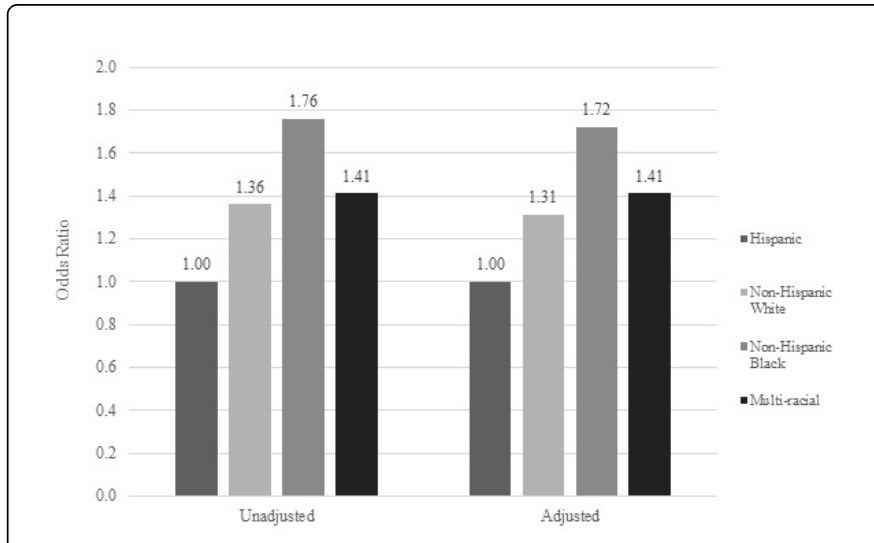

Figure 5: Unadjusted and adjusted associations between ethnicity and comorbidity (ASD and Epilepsy), National survey of children's health, 2012.

\begin{tabular}{|c|c|c|c|c|c|c|c|c|c|c|c|c|c|c|c|}
\hline \multirow[b]{2}{*}{ Race } & \multicolumn{3}{|c|}{ Unadjusted } & \multicolumn{2}{|c|}{ Model I } & & \multicolumn{2}{|c|}{ Model II } & & \multicolumn{2}{|c|}{ Model III } & & \multicolumn{2}{|c|}{ Model IV } & \multirow[b]{2}{*}{$p$} \\
\hline & aPOR & $95 \% \mathrm{Cl}$ & $\mathrm{p}$ & aPOR & $99 \% \mathrm{Cl}$ & $\mathrm{p}$ & aPOR & $99 \% \mathrm{Cl}$ & $P$ & aPOR & $99 \% \mathrm{Cl}$ & $\mathrm{p}$ & aPOR & $99 \% \mathrm{Cl}$ & \\
\hline Black/AA & 1.2 & $\begin{array}{l}0.64 \\
2.25\end{array}$ & 0.56 & 1.22 & $\begin{array}{l}0.53 \\
2.78\end{array}$ & 0.54 & 0.28 & $\begin{array}{l}0.02 \\
3.85\end{array}$ & 0.21 & 0.26 & $\begin{array}{l}0.02 \\
3.60\end{array}-$ & 0.19 & 0.26 & $\begin{array}{l}0.02- \\
3.64\end{array}$ & 0.19 \\
\hline Other & 0.89 & $\begin{array}{l}0.39 \\
1.63\end{array}$ & 0.71 & 0.92 & $\begin{array}{l}0.41 \\
2.03\end{array}-$ & 0.78 & 1.16 & $\begin{array}{l}0.44 \\
3.08\end{array}$ & 0.7 & 1.17 & $\begin{array}{l}0.44 \\
3.12\end{array}$ & 0.67 & 1.1 & $\begin{array}{l}0.41 \\
2.97\end{array}$ & 0.8 \\
\hline
\end{tabular}

Table 3: Association between ASD \& epilepsy co-occurrence and race, National survey of children's health, 2012.

Abbreviations, Notes, \& Model Descriptions: aPOR=adjusted prevalence odds ratio, $\mathrm{CI}=$ confidence interval, $\mathrm{p}=$ probability value. Unadjusted: Base model that examined Autism Spectrum Disorder/epilepsy co-occurrence and race. Model I: Adjusted for age and sex as clinically relevant variables. Model II: adjusted for age, sex, poverty, maternal education, and paternal education. Model III: adjusted for age, sex, poverty, maternal education, paternal education, and insurance. Model IV: adjusted for age, sex, poverty, maternal education, paternal education, insurance, and intellectual disability.

\section{Discussion}

Autism spectrum disorder (ASD) has been increasing in terms of prevalence and incidence in the United States due in part to the changes in diagnostic criteria. A public health challenge remains in terms of reducing the incidence of this condition or preventing complications including co-occurrence with other conditions, such as epilepsy. This study was proposed to examine the relationship between epilepsy and ASD in terms of causal pathway as to whether or not epilepsy leads to ASD or the reciprocal. Additionally, if such a pathway is established, or the comorbidity incidence is remarkable, this study aimed to assess the racial differences in the co-occurrence of ASD and epilepsy, as well as the risk markers for the co-occurrence.
We examined the prevalence of ASD, epilepsy, and the cooccurrence of ASD and epilepsy, as well as the racial differences in this prevalence. Secondly, we identified the risk markers for the cooccurrence. There are a few interesting findings in our study. First, among children in the United States, the prevalence of ASD is higher than epilepsy, while the co-occurrence of these conditions is lowest with respect to prevalence. Secondly, while epilepsy is higher among black children relative to whites, ASD is higher among whites. Thirdly, the co-occurrence of epilepsy and ASD prevalence is higher among blacks compared to whites. Fourthly, the risk markers for the cooccurrence were intellectual disability, maternal education, poverty as well as access to care as indicated by health insurance coverage. Finally, the racial differences in the co-occurrence were explained in party by 
social determinants of health namely maternal education, paternal education, and poverty.

We have demonstrated that epilepsy prevalence is higher among black children in a representative sample of United States children. These data support the findings from previous studies that observed racial disparities among those with epilepsy $[16,17]$. We examined the risk markers for epilepsy in an attempt to explain the racial variances in the prevalence. In the crude and unadjusted prevalence estimate, African American/black children compared to whites were two times as likely to be diagnosed with epilepsy. We built a multivariable model in an attempt to examine the racial variance therein. With this model building, racial differences did not persist after addressing and controlling for the confounding variables, namely insurance coverage, intellectual disability, poverty, maternal education, paternal education and body mass index. Consequently, based on our sample data, which represents a largely population of U.S. children, the racial differences in epilepsy prevalence between black and white children could be explained by racial variance in intellectual disability, access/utilization of health services (insurance coverage), social disadvantage as expressed by federal poverty line, maternal and paternal education (social disadvantage) as well as weight management among children. While the factors observed by our data could lead to a reduction in epilepsy prevalence as well as health inequity therein, these factors, to a greater extent, go beyond the medical models of disease etiology. The Epilepsy Foundation requires a larger collaboration with social services programs, insurance providers, the healthcare system and the U.S. Department of Health and Human Services. This collaboration is expected to result in preventative service coordination as well as healthcare system navigation.

ASD was shown in our sample to be higher among white children. This current finding supports several previously published data that examine ASD among children stratified by race and ethnicity $[18,19]$. The observed higher prevalence of ASD among white children relative to blacks may be explained in part by appropriate diagnoses of ASD in whites, in contrast to blacks who may rather be diagnosed with attention deficit disorder (ADD) [20,19]. Additionally, black children may have less access to the healthcare system and may therefore be under-diagnosed with ASD [20]. We also surmised that the increasing prevalence of congenital anomalies among white children may contribute to the higher prevalence of ASD in whites, given the possibility of uterine environmental insult $[3,11]$. In the crude and unadjusted prevalence estimate, black children were less likely to be diagnosed with ASD. We examined the risk markers for ASD and adjusted for them in the racial variability in ASD prevalence. Remarkably, after controlling for insurance, intellectual disability, poverty, maternal education, paternal education and obesity, the racial differences between blacks and whites in the prevalence of ASD did not persist. While the factors observed by the current study may contribute to reduction in ASD prevalence as well as health inequity therein, these factors to a greater extent go beyond the medical models of disease understanding and management. The National Autism Association, Autism Research Institute, and Autism Society require a larger collaboration with social services programs, insurance providers, the healthcare system and the U.S. Department of Health and Human Services in an attempt to better understand the complex etiologic and management pathways in this condition.

Whereas ASD was more common among white children, epilepsy was more common among blacks, but the co-occurrence of these conditions as comorbidity was higher among black children. We observed an increase in the odds of co-occurrence of these conditions in blacks relative to white children. There are no previous studies in this direction, explaining therefore our inability to either confirm or refute our findings based on published data. The factors predisposing to these conditions were examined. Our data indicated that the type of insurance as well as intellectual disability is associated with higher prevalence of the co-occurrence among blacks relative to white children. Insurance coverage which is a proxy to healthcare access and care utilization impacts morbidity and mortality across the life cycle [21]. We would therefore expect that the observed variance in ASD and epilepsy co-occurrence, even though imprecise in our adjusted data, results in part from the lower proportion of African American/ black children with private insurance relative to whites. Intellectual disability was another independent predictor of the co-occurrence. While no data exists to refute or affirm our claim in this vein, it is plausible that intellectual disability, which has a neurologic basis, contributes to epilepsy, ASD, as well as the co-occurrence of these conditions [11], however, intellectual disability may be a manifestation of co-occurrence implying complexities of causal inference.

Maternal education and poverty were associated with cooccurrence, and black/AA children were more likely to be associated with low maternal education and poverty. Adjusting for these factors as confounding in the association between race and co-occurrence did reduce the risk of this condition among blacks. Since the risk for the co-occurrence was higher among children with low maternal education and poverty, and because blacks/AA were disproportionately affected by these two potent predictors, balancing these factors across race is expected to influence or modify the point estimate or effect size for ASD-epilepsy co-occurrence. The current study observed precisely this modification, implying that addressing poverty and maternal education contributes to the reduction in ASD-epilepsy co-occurrence prevalence among black children in the United States.

Although we were unable to establish the causal relationship between epilepsy and autism, it is neurologically plausible to imagine the shared patho-physiology in ASD as well as epilepsy. Epilepsy has been shown, despite an unknown specific mechanism, to involve the hyper firing of the cortical neurons [6]. The electroencephalogram obtained in ASD tends to have similar appearance with that in epileptic seizures $[13,14]$. Our data unfortunately precludes our ability to examine the causal pathway as to the direction of the co-occurrence. The questions remain: does the presence or diagnosis of ASD predispose to the development of epilepsy? or does epilepsy or epileptic seizures result in the development of ASD? Without the answers to these questions, the causal mechanisms remain questionable, implying the need for prospective, preclinical data before non- experimental studies.

Despite the strengths of this study, mainly adequate sample size implying the statistical power of the study, and the sample representativeness meaning inherent generalizability, as well as the unique risk modeling approach to exposure effect, there are some limitations. First, as a cross-sectional design, we were unable to establish a clear temporal sequence with respect to the causal association between epilepsy and ASD as observed earlier. Secondly, despite controlling for the potential confounding, there are other variables that could confound the relationship between race and cooccurrence which were not available in the dataset-unmeasured confounding. Thirdly, despite adjusting for the potential confounding, unmeasured and residual confounding might have influenced the results of this study. However, it is highly unlikely to assume that our 
Citation: Holmes LJ, Stalnaker L, Casini J, Morgan I, John V, et al. (2017) Childhood Autism Spectrum Disorder and Epilepsy Co-occurrence: Sub-population Prevalence Variances and Risk Modeling. Epilepsy J 3: 1000119. doi:10.4172/2472-0895.1000119

Page 8 of 8

findings are driven solely by unmeasured or residual confounding. Nevertheless, no matter the statistical software used to control for confounding, residual confounding remains [22].

\section{Conclusion}

In summary, racial disparities exist in ASD-epilepsy co-occurrence prevalence, which did not persist after controlling for potential confounders; while public insurance, maternal education, poverty and intellectual disabilities remain potent predictors of the co-occurrence. These findings are suggestive of cautious optimism in assessing the causal direction in the co-occurrence of ASD and epilepsy among children, given the cross-sectional nature of the design used in this study. Therefore while caution should be exercised in the application of these findings in risk-adapted intervention mapping for risk reduction, effort to address racial disparities in ASD-epilepsy co-occurrence requires a careful consideration of health inequities as exposure function of health disparities in these conditions.

\section{Acknowledgements and Conflict of Interest}

The authors would like to thank Mrs. Patricia Oceanic and Ms. Yolanda Toole for coordinating and supporting the 2016 Health Equity Summer Scholars. Additionally, we thank Ms. Lavisha McClarin and Ms. Adrienne Wallace for critical review of the final manuscript. All authors have declared no conflict of interest.

\section{References}

1. Sutcliffe JS, Delahanty RJ, Prasad HC, McCauley JL, Han Q, et al. (2005) Allelic heterogeneity at the serotonin transporter locus (SLC6A4) confers susceptibility to autism and rigid-compulsive behavior. Am J Hum Genet. 77: 265-279.

2. Losh M, Adolphs R, Poe MD, Couture S, Penn D, et al. (2009) Neuropsychological profile of autism and the broad autism phenotype. Arch Gen Psychiatry 66: 518-526.

3. National Institute of Neurological Disorders and Stroke (NINDS) (2009) Autism Fact Sheet.

4. Viscidi EW, Johnson AL, Spence SJ, Buka SL, Morrow EM, et al. (2014) The association between epilepsy and autism symptoms and maladaptive behaviors in children with autism spectrum disorder. Autism 18: 996-1006.

5. Takano $T$ (2015) Interneuron dysfunction in syndromic autism: recent advances. Dev Neurosci 37: 467-475.

6. Bromfield EB, Cavazos JE, Sirven JI (2006) Chapter 1: Basic Mechanisms Underlying Seizures and Epilepsy. In: Bromfield EB, Cavazos JE, and Sirven JI (eds). An Introduction to Epilepsy. West Hartford, CT: American Epilepsy Society, pp: 1-26.
7. St Germaine-Smith C, Liu M, Quan H, Wiebe S, Jette N (2011) Development of an epilepsy-specific risk adjustment comorbidity index. Epilepsia 52: 2161-2167.

8. Center for Disease Control and Development (CDC) (2015) Epilepsy: Fast Facts. Available at: http://www.cdc.gov/epilepsy/basics/fast-facts.htm (accessed 27 July 2015).

9. Centers for Disease Control and Development (CDC) (2015) Autism Spectrum Disorder (ASD): Data and Statistics. Available at: http:// www.cdc.gov/ncbddd/autism/data.html (accessed 27 July 2015).

10. Hara H (2007) Autism and epilepsy: a retrospective follow-up study. Brain Dev 29: 486-490.

11. Wegiel J, Kuchna I, Nowicki K, Imaki H, Wegiel J, et al. (2010) The neuropathology of autism: defects of neurogenesis and neuronal migration, and dysplastic changes. Acta Neuropathol 119: 755-770.

12. Bolton PF, Carcani-Rathwell I, Hutton J, Goode S, Howlin P, et al. (2011) Epilepsy in autism: features and correlates. Br J Psychiatry 98: 289-294.

13. Oslejskova H, Dusek L, Makovska Z, Pejcochova J, Autrata R, et al. (2008) Complicated relationship between autism with regression and epilepsy. Neuro Endocrinol Lett 29: 558-570.

14. Gaitatzis A, Carroll K, Majeed A, Sander J (2004) The epidemiology of the comorbidity of epilepsy in the general population. Epilepsia 45: 1613-1622.

15. STATA Data Analysis and Statistical Software. Vers. 13.1. College Station, TX: Stata Corp LP, 2013. Computer software.

16. Kroner BL, Fahimi M, Kenyon A, Thurman DJ, Gaillard WD (2013) Racial and socioeconomic disparities in epilepsy in the District of Columbia. Epilepsy Res 103: 279-287.

17. Friedman D, Fahlstrom R (2013) Racial and ethnic differences in epilepsy classification among probands in the Epilepsy Phenome/Genome Project (EPGP). Epilepsy Res 107: 306-310.

18. Developmental Disabilities Monitoring Network Surveillance; Centers for Disease Control and Prevention (CDC) (2014) Prevalence of autism spectrum disorder among children aged 8 years-autism and developmental disabilities monitoring network, 11 sites, United States, 2010. MMWR Surveill Summ 63: 1-21.

19. Zaroff CM, Uhm SY (2012) Prevalence of autism spectrum disorders and influence of country of measurement and ethnicity. Soc Psychiatry Psychiatr Epidemiol 47: 395-398.

20. Mandell DS, Wiggins LD, Carpenter LA, Daniels J, DiGuiseppi C, et al. (2009) Racial/ethnic disparities in the identification of children with autism spectrum disorders. Am J Public Health 99: 493-498.

21. Biswas T, Walker P, Podder T, Efird JT (2015) Effect of Race and Insurance on the Outcome of Stage 1 Non-small Cell Lung Cancer. Anticancer Res 35: 4243-4249.

22. Holmes L Jr, Chan W, Jiang, Z, Du XL (2007) Effectiveness of androgen deprivation therapy in prolonging survival of older men with locoregional prostate cancer. J Prostate Cancer Prostatic Dis 10: 388-395. 